\title{
Cancer pharmacogenomics: do cancer cell lines have the right stuff?
}

\section{"With the advent of next-generation sequencing technologies, we are at a tipping point where we can gain unprecedented insights into the genetic alterations present in different cancers."}

\section{KEYWORDS: cancer = chemotherapy response $\approx \mathrm{NCl60}$ panel $\approx$ pharmacogenetics - tumor cell line}

In order to improve cancer patient outcomes, considerable effort has been undertaken to identify genomic alterations that impact patient response to chemotherapy. Once all the relevant alterations become known, it is hoped that the promise of individualized therapy, where each patient is treated with the optimal chemotherapy regimen for their tumor, would become fulfilled. Since clinical tissues are limited both in quantity and availability, many researchers have used cancer cell lines in their pharmacogenomics research. Cancer cell lines serve as useful models for clinical tumors because they proliferate readily, are experimentally tractable and are a renewable resource. One such resource in pharmacogenomics research is the NCI60 panel, a panel of 60 different cell lines encompassing nine different types of cancer (reviewed in [1]). The NCI60 panel has been utilized in studies to screen compounds for cytotoxic activity and potential clinical targets, while also identifying the necessary molecular factors required for cytotoxic activity. But with all the effort and money being put into pharmacogenomics research using cancer cell lines, it is appropriate to ask: how faithfully do cancer cell lines represent the tumors that they are being used to model?

First, do cancer cell lines look like the tumors they are modeling? The good news is that, in general, cancer cell lines look like their tissue of origin at the molecular level (i.e., geneexpression patterns and genomic alterations), at least when compared with cancer cell lines derived from other tissues. Using a microarraybased gene-expression profiling approach, Ross et al. were able to identify gene-expression patterns in the NCI60 cancer cell line panel that corresponded to the tissue of origin from which the cell lines were derived [2]. However, not all cancer cell lines fully represent their tissues of origin. A study by Sandberg et al. comparing expression profiles of the NCI60 panel with their corresponding in vivo tumors indicated that only approximately $50 \%$ of the cancer cell lines tested were most similar to their tissue of origin [3]. In fact, the authors found that the cancer cell lines varied widely in their expression of tumor characteristic genes, which impacted the propriety of some of the cell lines for the tissues they were supposed to be modeling. Some of the differences in gene expression between cancer cell lines and their tissue of origin are probably due to the presence of contaminating stromal cells in in vivo tumors; however, there is some evidence that stromal cells contribute little to the patterns of gene expression observed in tumors [4]. Overall, while many cancer cell lines fully represent their tissue of origin at the molecular level, care should be taken to determine the suitability of individual cancer cell lines for the research in question.

"...cancer cell lines are likely to be poor choices for discovering novel genomic or epigenetic alterations that are relevant to cytotoxic drug response..."

Next, do cancer cell lines behave similarly to the tumors they are intended to model to be useful for pharmacogenomics research? First, cancer cell lines are more appropriate for assessing the response to cytotoxic anticancer drugs, rather than the response to newer biologic agents which exert their anti-tumor effects via mechanisms other than eliciting cell death. Second, an important consideration to keep in mind when using cancer cell lines for pharmacogenomics research is that cell lines are generally more sensitive to cytotoxic agents than solid tumors [4]. Using a serial analysis of gene expression (SAGE)

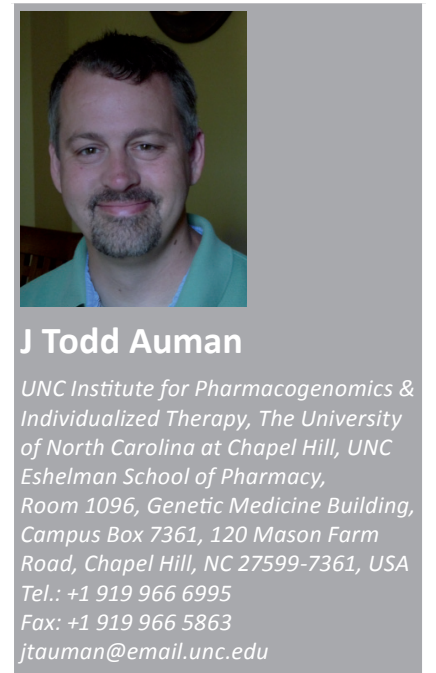

future medictiol $_{\text {fsg }}$ 
approach, Stein et al. was able to attribute this increased sensitivity to the fact that cancer cell lines exhibit elevated levels of expression of genes involved in protein and ribosome synthesis, while solid tumors exhibit elevated levels of expression of genes involved in immune response, the complement pathway and extracellular matrix proteins [4]. Another important question is: how well does testing in cancer cell lines predict responses in clinical trials with real world patients? When assessing whether there is a correlation between drug activity in Phase II clinical trials and preclinical activity in cancer cell line models, one study found that preclinical activity did not correlate with Phase II response, with the exception of non-small-cell lung cancer [5]. However, it does appear that compounds are likely to achieve some activity in a Phase II clinical trial if they have some activity in at least a third of the cells in the cell line panel [5]. Overall, cell line panels appear to perform relatively well for predicting whether a cytotoxic agent will work in a particular cancer type, but they do not seem to perform well when used to predict response within tumors of the same type, again with the exception of non-small-cell lung cancers [6]. Thus, cancer cell line panels appear to be appropriate for determining which types of cancers a cytotoxic agent is likely to elicit a response from, while they are generally not useful for predicting which patients will respond to a cytotoxic agent.

"...cancer cell lines still serve as a useful resource for studying the effects of specific genomic alterations identified in tumors."

\section{What is different between cancer cell lines \& clinical tumors?}

It is becoming more and more apparent that the process of culturing cells in vitro alters the genetic make-up of the cancer cell lines. For one thing, as mentioned earlier, cancer cell lines exhibit gene-expression profiles that are reflective of their selection for rapid growth [4], and further evidence suggests that growth under cell-culturing conditions leads to a transcriptional profile of increased nucleotide metabolism and RNA production [7]. This might be a consequence of always being in the presence of growth factors and other mitogens, which are components of most culture media [7]. Besides alterations at the transcriptome level, cancer cell lines also show genomic variations that are different to their tissue of origin. The result of multiple passages in culture is known to elicit genomic alterations [8], and the results of a recent study using array comparative genomic hybridization in breast cancers to assess the genomic alterations between tumors and cancer cell lines indicated that cell lines preserve the genomic alterations inherent in tumors, but they also carry secondary alterations that appear to be common to the cell-culturing process because they are found in multiple cell lines regardless of the tissue of origin [9]. Similarly, cancer cell lines were also found to have different epigenetic modifications compared with their tissues of origin. Cancer cell lines exhibited a higher level of methylated CpG islands compared with their respective tumors, with the same sites being methylated in both cancer cell lines and tumors, but with additional methylated sites, which seem to be a result of the cell-culturing process, observed in the cell lines [10]. These results suggest that cancer cell lines are likely to be poor choices for discovering novel genomic or epigenetic alterations that are relevant to cytotoxic drug response, since a large proportion of the genomic alterations are secondary to the cell-culturing process. However, these results do suggest that cancer cell lines still serve as a useful resource for studying the effects of specific genomic alterations identified in tumors.

There is one more reason that cancer cell lines are not favored for discovering novel factors involved in drug response. It is becoming apparent that in addition to increased sensitivity, cancer cell lines exhibit less cell line-cell line variation in sensitivity to cytotoxic agents than what is seen in tumors from patients [4]. For instance, the genomic alterations that are present in the NCI60 cell line panel, as compared with patientderived tumor tissue, suggest that the panel is only exploring a portion of the drug-sensitivity landscape [11]. Several studies examining breast cancers have come away with the similar conclusion that while the existing breast cancer cell lines are appropriate models for some of the breast cancer molecular subtypes, additional cancer cell lines are needed to fully capture the genomic diversity of breast cancers [12,13]. In addition, it was also recently postulated that existing colorectal cancer cell lines do not adequately reflect the molecular heterogeneity of clinical tumors [14]. Again, cancer cell lines may not be the best option for discovering novel genomic factors influencing cytotoxic drug response.

Therefore, to truly gain novel insights into chemotherapy response, the current cancer cell line models need to be supplanted by additional cell lines or studies involving clinical cancer tissues. With the advent of next-generation 
sequencing technologies, we are at a tipping point where we can gain unprecedented insights into the genetic alterations present in different cancers. While cancer cell lines may not be appropriate for the forefront of novel discoveries, they do play a critical role owing to their experimental history and tractability in gaining insight into the role that key genes discovered in clinical tumors play in chemotherapy response.

\section{Financial \& competing interests disclosure}

Dr Auman is supported in part by a GI SPORE grant from the NCI (P50-CA106991). The author has no other relevant affiliations or financial involvement with any organization or entity with a financial interest in or financial conflict with the subject matter or materials discussed in the manuscript apart from those disclosed.

No writing assistance was utilized in the production of this manuscript.

\section{Bibliography}

1 Shoemaker RH: The NCI60 human tumour cell line anticancer drug screen. Nat. Rev. Cancer 6(10), 813-823 (2006).

2 Ross DT, Scherf U, Eisen MB et al.: Systematic variation in gene expression patterns in human cancer cell lines. Nat. Genet. 24(3), 227-235 (2000).

3 Sandberg R, Ernberg I: Assessment of tumor characteristic gene expression in cell lines using a tissue similarity index (TSI). Proc. Natl Acad. Sci. USA 102(6), 2052-2057 (2005).

4 Stein WD, Litman T, Fojo T, Bates SE: A Serial Analysis of Gene Expression (SAGE) database analysis of chemosensitivity: comparing solid tumors with cell lines and comparing solid tumors from different tissue origins. Cancer Res. 64(8), 2805-2816 (2004).

5 Johnson JI, Decker S, Zaharevitz D et al.: Relationships between drug activity in NCI preclinical in vitro and in vivo models and early clinical trials. Br. J. Cancer $84(10)$, 1424-1431 (2001).
6 Voskoglou-Nomikos T, Pater JL, Seymour L: Clinical predictive value of the in vitro cell line, human xenograft, and mouse allograft preclinical cancer models. Clin. Cancer Res. 9(11), 4227-4239 (2003).

7 Ertel A, Verghese A, Byers S, Ochs M, Tozeren A: Pathway-specific differences between tumor cell lines and normal and tumor tissue cells. Mol. Cancer 5(1), 55 (2006).

8 Mamaeva SE, Kwang WJ: Karyotypic evolution of cells in culture: a new concept. Int. Rev. Cytol. 178, 1-40 (1997).

9 Tsuji K, Kawauchi S, Saito S et al.: Breast cancer cell lines carry cell line-specific genomic alterations that are distinct from aberrations in breast cancer tissues: comparison of the CGH profiles between cancer cell lines and primary cancer tissues. BMC Cancer 10(1), 15 (2010).

10 Smiraglia DJ, Rush LJ, Fruhwald MC et al.: Excessive $\mathrm{CpG}$ island hypermethylation in cancer cell lines versus primary human malignancies. Hum. Mol. Genet. 10(13), 1413-1419 (2001).
11 Szakács G, Gottesman MM: Comparing solid tumors with cell lines: implications for identifying drug resistance genes in cancer. Mol. Interv. 4(6), 323-325 (2004).

12 Kao J, Salari K, Bocanegra M et al.: Molecular profiling of breast cancer cell lines defines relevant tumor models and provides a resource for cancer gene discovery. PLoS One 4(7), E6146 (2009).

13 Lacroix M, Leclercq G: Relevance of breast cancer cell lines as models for breast tumours: an update. Breast Cancer Res. Treat. 83(3), 249-289 (2004).

14 Auman JT, McLeod HL: Colorectal cancer cell lines lack the molecular heterogeneity of clinical colorectal tumors. Clin. Colorectal Cancer 9(1), 40-47 (2010). 